\title{
Role of Self Expandable Drug Eluting Stent in Patients with Atypical Coronary Anatomy Short and Mid Term Clinical Outcome
}

M.H.Elemary ${ }^{1}$, A.I.Attia ${ }^{1}$, S.M.Abdou ${ }^{2}$, M.M.Ali $^{1}$ and S.A.Ahmed ${ }^{1}$

${ }^{1}$ Cardiovascular Dept., Faculty of Medicine, Benha Univ., Benha, Egypt

${ }^{2}$ Cardiovascular Dept., National Heart Institute

E-Mail: Sayed12@gmail.com

\begin{abstract}
Despite the advances in new generation stents, the optimal stenting considers a challenge to deal with atypical coronary anatomy; such as stent sizing in large, ectatic or aneurysmal vessels; and tapered vessels. Inflatable expandable stents are rounded and can only with significant effort oblige the variety in vessel; thrombotic vessels increment the danger of distal embolization and no-reflow; positive redesigning and vasodilation frequently bring about ensuing stent malapposition To survey the wellbeing, viability and practicality of self-expandable medication eluting stent in patients with atypical coronary life structures. This investigation was a clinical preliminary included 100 patients admitted to Saud Al-Babtain Cardiac Center (SBCC), Kingdom of Saudi Arabia (KSA) and Benha University emergency clinics, Arab Republic of Egypt (ARE) from January 2016 to December 2018 gave constant stable angina and intense coronary condition (STEMI, NSTEMI and USA) with their coronary angiography indicated all over again anomalous coronary life structures (ectatic, aneurysmal and additionally tightened vessel) with noteworthy coronary stenosis appropriate for PCI. The examination indicated that there were (53\%) STEMI patients followed by stable angina (21\%), NSTEMI (15\%), unsteady angina (10\%) and late MI (1\%). There were 42 and 58 patients with ectatic and tightened coronary life systems individually. With respect to result, no announced demise, MI, CABG in clinic, one month and a half year development. The clinically DTLR, there were one patient just had target sore MI, however we discovered this patient resistant he halted his double antiplatelet drug In This examination we found that the utilization of self-expandable stent is sheltered in patients gave steady and intense coronary disorder with atypical coronary life systems in regards to (in emergency clinic remain, short and mid-term) clinical result.
\end{abstract}

Keywords: Self-expandable stent, Atypical coronary artery, Ecstatic vessels aneurysmal vessels and tapered vessels.

\section{Introduction}

Throughout the previous 3 decades, there have been significant advances in nonsurgical coronary revascularization, and percutaneous coronary intercession (PCI) has now become the most generally utilized revascularization method around the globe. Albeit a considerable piece of this development has been the result of the all around reported improvement in results of patients with the expansive range of intense ischemic conditions who experience PCI versus clinical treatment [1].

Thought of the objectives of PCI must consider the depiction of what might be viewed as "ideal PCI." The innovative insurgency, overwhelmed by stent implantation and advances in antithrombotic and antiplatelet treatments, is persistently developing. As an immediate result, there has been an emotional decline in the requirement for critical $\mathrm{CABG}$ medical procedure following PCI on the grounds that the issue of intense or compromised conclusion has been nearly dispensed with $[2,3]$.

Coronary course aneurysm is a generally extraordinary confusion described by coronary vein dilatation with width that surpasses half of the ordinary neighboring portions with atherosclerosis being the most widely recognized etiology [4].

Stent malapposition has been connected with stent thrombosis [5]. As a stent is extended with high weight, quick injury happens somewhere down in the vessel divider inside the stented portion just as in the unscaffolded diligent edges. Critically, a few stent preliminaries, particularly those including brachytherapy, have caused new to notice the issue of quickened lumen misfortune at stent edges, which represents up to $33 \%$ of target vessel revascularization in patients treated with swell expandable stents [6].

Self-expandable coronary stent framework intended to permit ideal adjustment of the stent swaggers to the vessel divider so as to lessen the event of stent malapposition that is viewed as one of the most impressive morphometric indicators of stent thrombosis $[7,8]$, progressively over this sort of stent possibly reasonable for subgroup of coronary injuries with aneurysm and/or disparity in measurement among stenotic and widened portions proximal or distal to the coronary sore .

\section{Aim of the work}

The aim of this study was to assess the safety, efficacy and feasibility of self-expandable drug eluting stent in patients with atypical coronary anatomy .

\section{Patients and methods}

This is an imminent report included 100 patients admitted to Saud Al-Babtain Cardiac Center (SBCC), Kingdom of Saudi Arabia (KSA) and Benha University emergency clinics, Arab Republic of Egypt (ARE) from January 2016 to December 2018 gave incessant stable angina and intense coronary disorder (STEMI, NSTEMI and USA) with their coronary angiography indicated once more strange coronary life structures (ectatic, aneurysmal and additionally tightened vessel) with critical coronary stenosis appropriate for PCI. 


\section{Exclusion Criteria}

- Previous stenting of the target lesion (in-stent restenosis).

- Contraindication to aspirin or clopidogril or ticagrelor.

- Female gender during pregnancy.

- Patients with significant (moderate or severe) valvular heart disease.

- Patients with pericardial disease (constrictive pericarditis).

- Patients with recent stroke or transient ischemic attack.

- Life expectancy less than 6 months due to any clinical situation .

\subsection{Methods}

- All patients in the study were subjected to the following:

- Informed consent.

- Thorough history taking and general examination.

Stressing on DM, hypertension, dyslipidemia, smoking, family history of ischemic heart disease, symptoms of heart failure and symptoms of ischemic heart disease wither acute or chronic .

\section{- Full cardiac examination}

Especially for any signs of acute heart failure or advanced comorbidity.

\section{- Laboratory investigations}

All included patients were subjected to biochemical tests including LDL-C, HDL-C, total cholesterol , triglyceride ,serum urea and creatinine, and blood sugar test to evaluate for risk factors and other comorbidity . Cardiac troponin, total CK and CK MB : to detect NSTEMI or STEMI .

\section{- Electrocardiogram}

1. To assess any significant arrhythmia .

2. Significant ST segment elevation to detect STEMI .

3. Dynamic ST -T wave changes to detect USA .

4. Pathological Q wave in case of previous MI .

\section{- Echocardiography}

Assessment was performed and the Left ventricular end-systolic volume, end-diastolic volume and LV ejection fraction were measured using Simpson method and to detect if there is any significant LV systolic dysfunction, pericardial disease or significant valvular heart disease from parasternal and apical two and four chamber views, standard views according to recommendations of American Society of Echocardiography. Also assessment of resting wall motion abnormality as (hyperkinesia, akinesia, or dyskinesia, scar, aneurysm) of cardiac walls to detect ischemia [9].

- Coronary angiography

- The diagnostic coronary angiography procedure was performed using a Philips Allura Xper FD 10/10 biplane cardiovascular X-ray system. $\circ$ The vascular approach depends on the operators decision .

- Coronary angiogram was done using the standard views of the center.

\section{- Angiographic Assessments}

Quantitative coronary angiography ( QCA ) was performed using dedicated soft ware ( Q Angio X A version 7.3 :Medis leiden the Netherlands )

Pre and post procedural reference vessel diameter (RVD) , minimal luminal diameter (MLD) , and percentage diameter stenosis ( \% DS ) and lesion length were obtained .

Angiographic success was defined as final residual stenosis of $>20 \%$ by offline QCA , and TIMI III flow on the final angiogram

An optimal results was defined as $>10 \%$ residual stenosis of the target lesion, without angiographically visible dissection at the edge of the stent and or the side branch with TIMI III flow in both vessels [10].

\section{Stent deployment and Devices used}

The investigational device used in this study was the Self-Apposing Coronary Stent System (STENTYS SA) in 48 patients and ( $\mathrm{X}$ position DES ) in 52 patients . It is made from nitinol (a nickel-titanium alloy) with a nominal strut width of $68 \mu \mathrm{m}$ (0.0027 in), compatible with a $6 \mathrm{~F}$ guide catheter.

It is available in 3 lengths $(17 \mathrm{~mm}, 22 \mathrm{~mm}$ and $27 \mathrm{~mm})$, and in three diameter sizes: small $(2.5-3.0 \mathrm{~mm})$, medium $(3.0-3.5 \mathrm{~mm})$, and large $(3.5-4.5 \mathrm{~mm})$. The strut thickness is 102 microns (small size) or 133 microns (medium and large sizes). The large self expandable stent can expand over $6.0 \mathrm{~mm}$ suitable for absolute vessel diameters of $>4.5 \mathrm{~mm}$.

Recommended vessel diameter is the indicated vessel diameter for the stent size to ensure sufficient radial force and minimal foreshortening. Stents can grow up to the maximum vessel diameter in case of ectatic or tapered vessels.

The distal vessel diameter was used to determine the Stentys stent diameter : At the stent diameter range boundaries, it is recommended to use the smaller size . For example, a vessel diameter of $3.5 \mathrm{~mm}$ RVD would receive a 3.0- $3.5 \mathrm{~mm}$ Stentys stent not a 3.5-4.5 $\mathrm{mm}$ one [11] .

\section{Follow up scheme}

Subjects were followed up for one month , 3 months and 6 months .

\section{Study end points}

- Primary end point:

1. TIMI flow grade 3 distal to the target lesion .

2. Residual stenosis less than $10 \%$.

- Secondary end point:

Patients were evaluated clinically during hospital stay, one, 3 and six months after procedure regarding major adverse cardiac events 


\section{Cardiac death}

Cardiac death includes death resulting from an acute myocardial infarction (MI), sudden cardiac death, death due to heart failure (HF), or death due to cardiac procedures.

\section{Target lesion MI .}

When there is evidence of myocardial necrosis in a clinical setting consistent with myocardial ischemia which cannot be explained by another native coronary.

3. CABG.

Coronary artery bypass surgery to the target vessel performed for restenosis or other complication involving the target lesion

4. Clinically driven target lesion revascularization .

Any repeat percutaneous intervention of the target lesion (including $5 \mathrm{~mm}$ proximal and $5 \mathrm{~mm}$ distal to the target lesion) of the target vessel performed for restenosis or other complication involving the target lesion.

Revascularization is clinically driven if the target lesion diameter stenosis is $>50 \%$ by quantitative coronary angiography (QCA) and the subject has clinical or functional ischemia which cannot be explained by another native coronary or bypass graft lesion. Clinical or functional ischemia includes any of the following:

a. A history of angina pectoris, presumably related to the target vessel .

b. Objective signs of ischemia at rest (electrocardiographic changes) or during exercise test (or equivalent), presumably related to the target vessel .

c. Abnormal results of any invasive functional diagnostic test e.g., fractional flow reserve (FFR) .

\section{Results}

This study was conducted on 100 patients underwent PCI using self expandable stent in SBCC, KSA and Benha University hospitals in the period from January 2016 to December 2018.

\section{Demographic and risk factors}

The mean age of patients in this study was 53 years with standard deviation of 13 years with age range from 25 to 87 years , $95 \%$ of the patients were males, $60 \%$ of patients were smokers, $23 \%$ were ex-smokers and 17 patients never smoke, we found $39 \%$ diabetic $10 \%$ IDDM and $29 \%$ NIDDM, there were $52 \%$ were hypertensive, $22 \%$ dyslipidemia, , $1 \%$ had prior CVA , $7 \%$ had renal impairment, there were $1 \%$ of patients had history of peripheral vascular disease, patients with history of previous PCI was $17 \%$ and $4 \%$ had prior $\mathrm{CABG}$. Positive family history recorded in $15 \%$ of patients included in this study.

\section{Clinical presentation}

The ST segment elevation myocardial infarction was the main clinical presentation, it represented (53\%) followed by stable angina (21\%), NSTEMI (15\%), unstable angina $(10 \%)$ and late presentation MI (1\%).

\section{Thrombolytic therapy use}

Thrombolytic therapy was reported in $9 \%$ of patients' pre procedural.

\section{Type of procedure}

The most frequent type was emergency (41\%) followed by elective (34\%) then urgent $(25 \%)$.

\section{Coronary vessel affected}

The most frequent coronary artery affected was LAD $(53 \%)$ followed by RCA $(23 \%)$ and LCX $(20 \%)$ then LM (4\%).

\section{Type of segment affected}

The most frequent affected segment of the coronary artery was proximal segment $(58 \%)$ followed by mid segment $(34 \%)$ then distal segment $(8 \%)$.

\section{ACC/AHA classification and TIMI flow}

> As regard ACC/AHA classification, type C lesion was the most frequent lesion $(48 \%)$ followed by type B2 (30\%), type A (15\%) then type B1 (7\%).

$>$ As regard TIMI flow,

- TIMI flow pre procedure was 0 in $31 \%$ of patients, 1 in $28 \%$ of patients, 2 in $27 \%$ of patients and 3 in $14 \%$ of patients.

- TIMI flow post procedural was 3 for all patients.

\section{Vessel type}

In our study we found that patients with tapered vessels represented $58 \%$ while patients with ectasia represented $42 \%$

Pre and post procedural measures in the whole patients ( QCA )

$>$ RVD proximal

Mean RVD proximal was 3.7 with standard deviation of 0.6 .

$>$ RVD distal

Mean RVD distal was 3 with standard deviation of 0.5 .

$>$ MLD

Mean MLD was 0.59 with standard deviation of 0.34 .

$>$ Stenosis

Mean stenosis was $84 \%$ with standard deviation of $9 \%$. By QCA or visual estimation .

$>$ Lesion length

Mean lesion length was 22 with standard deviation was 4.9.

$>$ Stent type

$58 \%$ of stents were $\mathrm{x}$-position ( DES) type and $48 \%$ were stentys (type included 46\% DES and 2\% BMS) .

$>$ PDD proximal

Mean PDD proximal was 3.7 with standard deviation of 0.6 .

$>$ PDD distal

Mean PDD distal was 3 with standard deviation of 0.5 . 
Pre procedural measures in patients with tapered coronary arteries (QCA).

$>$ RVD proximal

Mean RVD proximal was $3.54 \mathrm{~mm}$ with standard deviation of $0.53 \mathrm{~mm}$.

$>$ RVD distal

Mean RVD distal was $2.87 \mathrm{~mm}$ with standard deviation of $0.45 \mathrm{~mm}$.

\section{$>$ MLD}

Mean MLD was $0.52 \mathrm{~mm}$ with standard deviation of $0.31 \mathrm{~mm}$.

$>$ Stenosis

Mean stenosis was $85.5 \%$ with standard deviation of 8.4 $\%$. By QCA or visual estimation

$>$ Lesion length

Mean lesion length was $22.2 \mathrm{~mm}$ with standard deviation was $5.4 \mathrm{~mm}$

Post procedural measures in patients with tapered coronary arteries ( QCA ).

$>$ PDD proximal

Mean PDD proximal was $3.54 \mathrm{~mm}$ with standard deviation of $0.53 \mathrm{~mm}$.

$>$ PDD distal

Mean PDD distal was $2.87 \mathrm{~mm}$ with stadard deviation of $0.45 \mathrm{~mm}$

Pre procedural measures in patients with ectatic coronary arteries (QCA).

$>$ RVD proximal

Mean RVD proximal was $3.9 \mathrm{~mm}$ with standard deviation of $0.6 \mathrm{~mm}$.

$>$ RVD distal

Mean RVD distal was $4.3 \mathrm{~mm}$ with standard deviation of $0.62 \mathrm{~mm}$.

$>$ MLD

Mean MLD was $0.69 \mathrm{~mm}$ with standard deviation of 1.1 $\mathrm{mm}$.

$>$ Stenosis

Mean stenosis was $82.64 \%$ with standard deviation of $8.87 \%$. By QCA or visual estimation .

$>$ Lesion length

Mean lesion length was $22.1 \mathrm{~mm}$ with standard deviation was $5 \mathrm{~mm}$.
Post procedural measures in patients with ectatic coronary arteries (QCA).

$>$ PDD proximal

Mean PDD proximal was $3.9 \mathrm{~mm}$ with standard deviation of $0.6 \mathrm{~mm}$.

$>$ PDD distal

Mean PDD distal was $4.3 \mathrm{~mm}$ with standard deviation of $0.61 \mathrm{~mm}$

\section{Type of antiplatelet used}

IC aggrastate was utilized in $49 \%$ of patients while IV aggrastate was utilized in $41 \%$ of patients., Aspirin was utilized in $100 \%$ of patients. Ticagrelor was utilized in $74 \%$ of patients. what's more, Clopidogril was utilized in $26 \%$ of patients when ticagrelor not accessible Short and middle of the road term Complications

Passing was not detailed in any patient in medical clinic, at multi month or at a half year. MI was accounted for in just 1 patient (1\%) at 3 months. No MI was accounted for at clinic or at half year likewise No crisis CABG was accounted for in all patients in emergency clinic, at multi month or at a half year, and clinically DTLR was accounted for in just a single patient (1\%) at 3 months because of stop of prescriptions.

This patient was 53 years of age hypertensive male conceded first time as NSTEMI with ectatic coronary conduits with thrombus in mid LCX-PDA injury desire done, prediltation and stenys 3-3.5 $* 22$ conveyed with post dilatation finished with $3.75 * 8$ HPB with $10-12-14$ ATM with no leftover stenosis a TIMI 3 post procedural ., after around 3 months understanding readmitted with STEMI after clinical assessment of this patient we found that patient was not taken his prescriptions which incorporate headache medicine and ticagrelor and we discovered there thrombus trouble over the recently sent stentys stent territory, goal and PTCA was finished with TIMI 3 stream post technique. quiet at that point followed up for 3 months and no inconveniences were accounted for identified with this examination Different methodology

$>$ different techniques utilized during intercession were intra vascular ultrasound IVUS was done in $17 \%$ of patients pre and post, Fractional stream hold FFR was done in $3 \%$ of patients, and Thrombus Aspiration was done in $21 \%$ of patients $11 \%$ in patients with ectatic vessel and the other $10 \%$ in patients with tightened vessel.

Table (1) Demographics and risk factors in the whole study population .

\begin{tabular}{|c|c|c|}
\hline Age (years) & Mean \pm SD & $53 \pm 13$ \\
\hline \multirow[t]{2}{*}{ Gender } & Females n $(\%)$ & $5(5.0)$ \\
\hline & Males n $(\%)$ & $95(95.0)$ \\
\hline \multirow[t]{3}{*}{ Smoking } & Yes $\mathrm{n}(\%)$ & $60(60.0)$ \\
\hline & Ex-smoker $\mathrm{n}(\%)$ & $23(23.0)$ \\
\hline & Never smoke $\mathrm{n}(\%)$ & $17(17.0)$ \\
\hline Diabetes mellitus & Yes $\mathrm{n}(\%)$ & $39(39.0)$ \\
\hline Hypertension & $\mathrm{n}(\%)$ & $52(52.0)$ \\
\hline Dyslipidemia & $\mathrm{n}(\%)$ & $22(22.0)$ \\
\hline Prior CVA & $\mathrm{n}(\%)$ & $1(1.0)$ \\
\hline
\end{tabular}


Table (1) Continue

\begin{tabular}{llll}
\hline Renal impairment & Yes & $\mathrm{n}(\%)$ & $7(7.0)$ \\
PVD & Yes & $\mathrm{n}(\%)$ & $1(1.0)$ \\
Prior PCI & Yes & $\mathrm{n}(\%)$ & $17(17.0)$ \\
Prior CABG & Yes & $\mathrm{n}(\%)$ & $4(4.0)$ \\
Positive family history & Yes & $\mathrm{n}(\%)$ & $15(15.0)$ \\
\hline
\end{tabular}

Table (2) Frequency distribution of coronary vessel affected.

\begin{tabular}{ll}
\hline & $\mathbf{n}(\%)$ \\
\hline LM & $4(4.0)$ \\
LAD & $53(53.0)$ \\
LCX & $20(20.0)$ \\
RCA & $23(23.0)$ \\
\hline
\end{tabular}

Table (3) Frequency distribution of type of vessel.

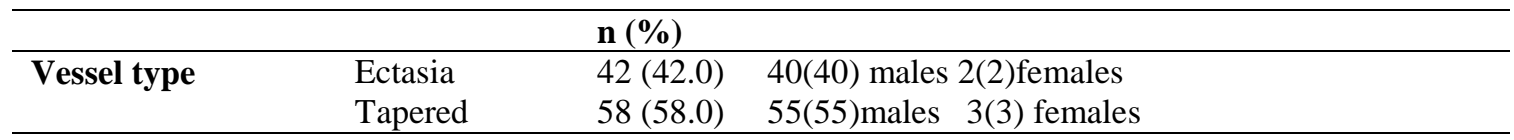

Table (4) Pre and post procedural measures in the whole patients(QCA).

\begin{tabular}{lcc}
\hline RVD proximal & Mean \pm SD & $\mathbf{3 . 7} \pm \mathbf{0 . 6}$ \\
\hline RVD distal & Mean \pm SD & $3 \pm 0.5$ \\
MLD $(\mathbf{m m})$ & Mean \pm SD & $0.59 \pm 0.34$ \\
Stenosis $(\%)$ & Mean \pm SD & $84 \pm 9$ \\
Lesion length & Mean \pm SD & $22 \pm 4.9$ \\
Stent type & Stentys & $48(48.0)$ \\
& X position & $52(52.0)$ \\
PDD proximal & Mean \pm SD & $3.7 \pm 0.6$ \\
PDD distal & Mean \pm SD & $3 \pm 0.5$ \\
\hline
\end{tabular}

Table (5) Pre and post procedural measures in patients with tapered coronary arteries ( QCA ).

\begin{tabular}{lll}
\hline RVD proximal & Mean \pm SD & $3.54 \pm 0.53$ \\
\hline RVD distal & Mean \pm SD & $2.87 \pm 0.45$ \\
MLD (mm) & Mean \pm SD & $0.52 \pm 0.31$ \\
Stenosis $(\%)$ & Mean \pm SD & $85.5 \pm 8.4$ \\
Lesion length & Mean \pm SD & $22.22 \pm 5.4$ \\
PDD proximal & Mean \pm SD & $3.54 \pm 0.53$ \\
PDD distal & Mean \pm SD & $2.87 \pm 0.45$ \\
\hline
\end{tabular}

Table (6) Pre and post procedural measures in patients with ectatic coronary arteries (QCA).

\begin{tabular}{lll}
\hline RVD proximal & Mean \pm SD & $\mathbf{3 . 9} \pm \mathbf{0 . 6}$ \\
\hline RVD distal & Mean \pm SD & $4.3 \pm 0.6$ \\
MLD (mm) & Mean \pm SD & $0.69 \pm 1.1$ \\
Stenosis $(\%)$ & Mean \pm SD & $82.64 \pm 8.87$ \\
Lesion length & Mean \pm SD & $21.1 \pm 5$ \\
PDD proximal & Mean \pm SD & $3.9 \pm 0.6$ \\
PDD distal & Mean \pm SD & $4.3 \pm 0.61$ \\
\hline
\end{tabular}

Table (7) Frequency distribution of in hospital, 1, 3 and 6 months complications.

\begin{tabular}{lll}
\hline & & n $(\%)$ \\
\hline Death & In-hospital & $0(0.0)$ \\
1 month & $0(0.0)$ \\
3 months & $0(0.0)$ \\
6 months & $0(0.0)$
\end{tabular}




\begin{tabular}{lll} 
Table (7) Continue & & \\
\hline MI & In-hospital & $0(0.0)$ \\
& 1 month & $0(0.0)$ \\
& 3 months & $1(1.0)$ \\
& 6 months & $0(0.0)$ \\
Emergency bypass & In-hospital & $0(0.0)$ \\
& 1 month & $0(0.0)$ \\
& 3 months & $0(0.0)$ \\
Clinically DTLR & 6 months & $0(0.0)$ \\
& In-hospital & $0(0.0)$ \\
& 1 month & $0(0.0)$ \\
& 3 months & $1(1.0)$ \\
& 6 months & $0(0.0)$ \\
\hline
\end{tabular}

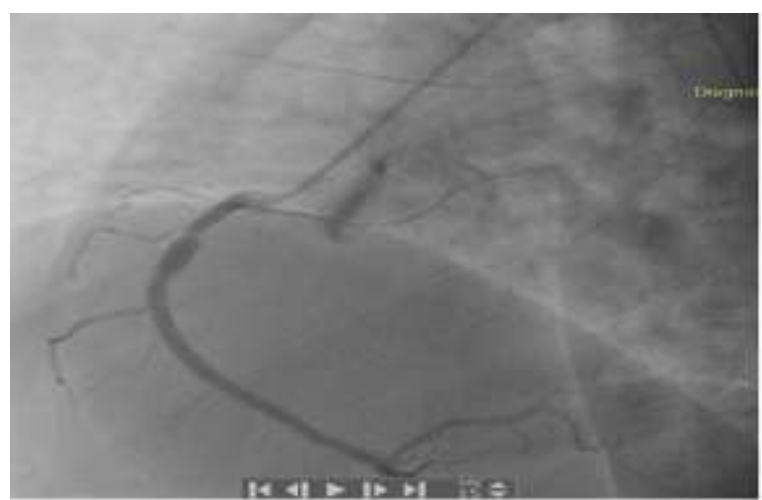

Fig (1) Ectatic RCA preintervention

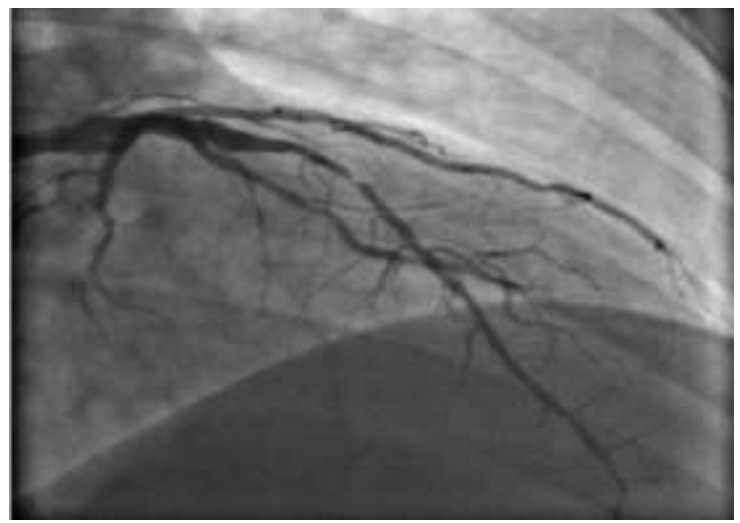

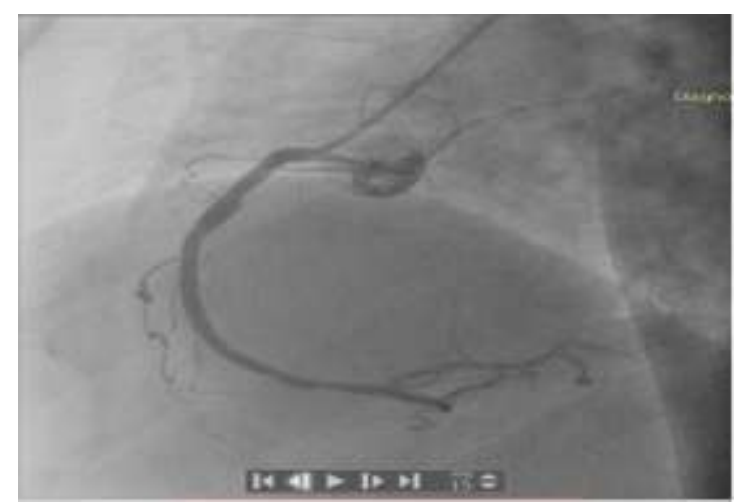

Fig (2) post intervention angiography in ectatic RCA

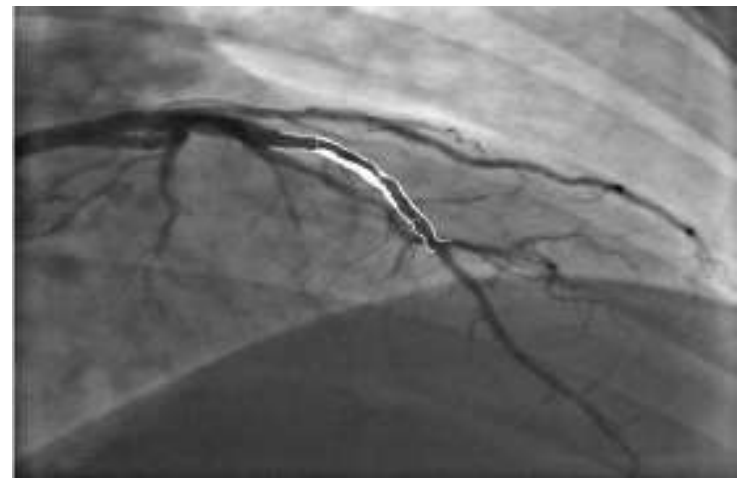

Fig (3) tapered LAD angiography.
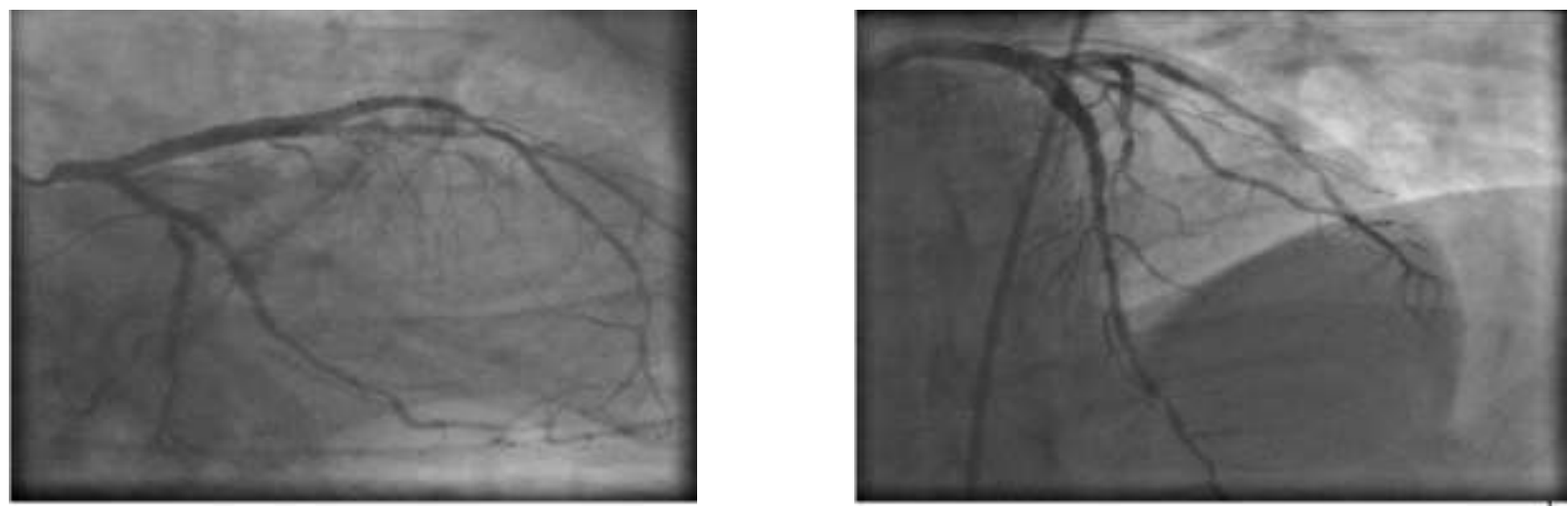

Fig (4) post intervention tapered LAD lesion . 


\section{Discussion}

This examination was done to assess the utilization of the self-expandable stent with the report the in emergency clinic, one month and half year clinical result in a gathering of patients' with atypical coronary life structures. The signs for self-expandable stent utilize was

coronary aneurysm, ectasia, tightening, outright vessel distances across.

One hundred patients were remembered for this examination and we found that self-expandable stent is sheltered and productive in patients with interminable stable angina and intense coronary condition with atypical coronary life systems.

\section{Atypical coronary life structures}

In our investigation it was discovered that patients with tightened vessels spoke to $58 \%$ and with ectasia spoke to $42 \%$.

While in study led by [18] for patients with complex coronary life structures they found that (33\%) and $(24 \%)$ )of patients had tightened and ectatic coronary supply route separately.

Likewise in study directed by [13], ( $50.4 \%$ ) of patients had ectatic coronary corridor, yet in study led by Luigi and his associate 2016(14), (15\%) of patients had ectatic coronary course. This past rate comparable to all inclusive community, so it is not the same as our rate.

\section{Clinical introduction}

This investigation found that the most regular sign was STEMI (53\%) trailed by stable angina (21\%), NSTEMI $(15 \%)$, temperamental angina $(10 \%)$ and late MI (1\%).

While In study directed by (12), patients introduced by stable angina was (39\%), NSTEMI was ( $46 \%)$, and $(16 \%)$ and for precarious angina .

Likewise in study led by Simona and his associate 2016(13), patients introduced by STEMI (59\%), stable angina (18\%) and NSTEMI ( $32 \%)$. What's more, in study led by (14) patients introduced by STEMI (30\%), Stable angina (half), and NSTEMI/USA (20\%)

In any case, in APPOSITION I study done by Giovann and his associate in 2011(15), APPOSITION II study done by ROBERT 2012(16), and his partner, APPOSITIN III examination by [17] and APPOSITION IV done by Robert and his (18) every single included patient introduced by STEMI

\section{Thrombolytic treatment}

With respect to treatment in this investigation, there were $9 \%$ of STEMI patients got thrombolytic treatment.

By surveying reads accomplished for self expandable coronary stent, no patient read for selfexpandable coronary stent named as got thrombolytic treatment at time of introduction .

\section{Sort of method}

Thirty four \% of patients was named as elective, 25 $\%$ as earnest and $41 \%$ as crisis in this investigation.
While in APPOSITION I study done by [15] APPOSITION II study done by [16] and his associate, APPOSITIN III investigation by [17] and APPOSITION IV done by (18) all patients marked as crisis.

\section{Target vessel}

In this examination we found the most incessant influenced vessel was LAD (53\%) trailed by RCA (23\%) and LCX (20\%) at that point LM (4\%).

While in study directed by (13) the influenced vessel was LAD (38.5\%), RCA (47.7\%), LCX (11.9\%) and LM $(0.9 \%)$.

What's more, in study led by (14) the influenced vessel was LAD (30\%), RCA (37.5\%), LCX (10\%) and LM $(17.5 \%)$.

While in (15) the influenced vessel was LAD (52\%), RCA (40\%), LCX (8\%)., (16) the influenced vessel was LAD (44.2\%), RCA (44.2\%), LCX (11.6\%)., (17), the influenced vessel was LAD (37\%), RCA (49\%), LCX (14\%)., (18) the influenced vessel was LAD (36.7\%), RCA (48.9\%), LCX (14.4\%) . LM not concentrated in the recently referenced investigations.

\section{Target portion of influenced vessel}

Concerning fragment influenced the most successive influenced portion was proximal (58\%) trailed by mid section (34\%) at that point distal fragment (8\%).

As far as anyone is concerned no different investigations with respect to self-expandable coronary stent named their patients in regards to fragment of influenced vessel.

ACC/AHA characterization of coronary injury

In this examination type $\mathrm{C}$ injury was found in (48\%) of patients, type B2 sore (30\%), type A sore $(15 \%)$ at that point B1 (7\%).

While in (17) patients named as type $\mathrm{C}$ sore seemed to be (24\%), type B2 was (44\%) and that for type A/B1 was $(33 \%)$.

\section{Pre and post procedural TIMI stream Pre procedural}

This investigation demonstrated that there was $(31 \%)$ of patients gave TIMI $0,(28 \%)$ with TIMI 1 , (27\%)with TIMI 2 and (14\%) of the patients with TIMI 3.

While in study led by (13), there was (38.5\%) of patients gave TIMI $0,(12.8 \%)$ with TIMI 1, (13.8\%)with TIMI 2 and (33.9\%) of the patients with TIMI 3.

Likewise in APPOSITION III investigation 2015 [17] patients named with TIMI 0 was (57\%), TIMI 1 (13\%), TIMI 2 (17\%) and TIMI 3 was (13\%). what's more, in APPOSITION IV(18) study 2016 patients named with TIMI 0 was $(51.7 \%)$, TIMI 1 (1.7\%), TIMI $2(22.5 \%)$ and TIMI 3 was (18\%).

This high rate in regards to preprecdural TIMI 0 follow in APPOSITION III(17) study might be because of that all patients remembered for this examination gave STEMI 


\section{Post procedural}

All patient in this examination had TIMI 3 stream post procedural.

While in study directed by (13) there was $(0.9 \%)$ of patients with TIMI 0 and TIMI $1,(2.7 \%)$ with TIMI 2 and $(96 \%)$ of the patients with TIMI 3 post method. There is distinction between this examination and our investigation results in regards to evaluations of TIMI stream, and this might be on the grounds that all patients in [13] study was given STEMI

In any case, in (17)patients named with TIMI 0 was (1\%), TIMI $1(0.4 \%)$, TIMI $2(4 \%)$ and TIMI 3 was $(94.5 \%)$. also, in (18) no patients marked with TIMI 0 or TIMI 1 yet patients with TIMI 2 was (14.4\%) and TIMI 3 was $(85.5 \%)$ post methodology . and furthermore this distinction in level of evaluation of TIMI stream conveyance might be because of that all patients was given STEMI in postulations examines

\section{Meds utilized}

In this examination the pre-owned medications was Aspirin in all patients, ticagrelor ( $74 \%)$ of patients, colopidogrel utilized in $(26 \%)$ of patients when ticagrelor not accessible.

IC and/or IV aggrastate in (41\%) of patients if there was visual doubt of enormous thrombus weight or remaining thrombus.

While in study directed by (13), during system utilization of aggrastate was accounted for in ( $30.3 \%)$ of examined patients.

Be that as it may, in [17] ibuprofen was utilized in $(97 \%)$, colopidogrel was utilized in (39\%), ticagrelor was utilized in ( $2 \%$ ), aggrastate was utilized in $(24 \%)$ of patients. furthermore, in [18] headache medicine was utilized in $(100 \%)$, colopidogrel was utilized in ( $31.1 \%$ ), ticagrelor was utilized in ( $68.9 \%)$, aggrastate was utilized in $(33.3 \%)$ of patients.

\section{Adjuvant techniques}

- In this investigation, IVUS utilized in (17\%) of patients which incorporate $(11 \%)$ ectatic and (6\%) tightened vessels.

While in APPOSITION I study 2011(15), IVUS was utilized pre and post procedural in all patients as a daily practice for study reason .

Thrombus yearning was done in this investigation in (21\%) of included patients 11 patients was with ectasia and 10 patients with tightened vessel.

Additionally in study led by Simona and his associate 2016(13), thrombus desire acted in (35.8\%) .

While thrombus yearning acted in (16), in (71.4\%), likewise in (17) in ( $74 \%$ ) and in (18)in (84.4\%) of patients .

Hight level of thrombus yearning in $(16,17,18)$ these was because of that every single remembered tolerant for the before referenced investigations was given STEMI and this isn't care for the patients remembered for our examination which incorporate just $53 \%$ of patients gave STEMI

FFR was utilized in (3\%) of patients.

\section{Clinical development}

- In this investigation no records with respect to death, MI, crisis CABG and crisis DTLR in regards to in clinic coarse and one month development. In half year follow up additionally no record for the upper referenced difficulties aside from (1\%) of patient had MI following multi month present strategy due on stop of meds .

Our outcomes were in concurrence with bring about examination done by Pastormerlo and Luigi 2016(14), they recorded that year and a half after the file maker patients was on single antiplatelet treatment, one patient created stent restenosis in the second OM of LCX branch with littlest stentys which need TLR utilizing PTCA in light of the fact that the vesele breadth is too little .

Comparative outcomes were introduced by APPOSION II study done by ROBERT 2012, and his associate (16), they detailed that no cardiovascular demise, MI, crisis CABG and clinically DTVR during the in medical clinic course and in, one month, however in half year follow up there were one patients(1/72) had clinically DTVR and this outcomes in no heart passing, $\mathrm{MI}$ and crisis CABG.

There was results announced In APPOSITION I study done by (15) they assessed patients with intense myocardial dead tissue got stentys self-expandable coronary stent.

They found that no cardiovascular demise, MI, crisis CABG and TVR during the in clinic coarse and in half year follow up with respect to heart passing, MI, crisis CABG.

While in same examination (15) there were $12 \%$ (3/25 patients ) had clinically DTVR with respect to a half year follow up .

Low rate results announced in ( APPOSITION III, 2015) concentrate by (17)they detailed that in 30 dayes result there were $(1.2 \%-\mathrm{n} 11 / 965)$ heart passing, MI (1.1\% - n 11/965), clinically DTLR (2.5\%-n 21/965

High rate related outcomes were accounted for by (12), they recorded that as long as a half year there were concurrence with present examination results yet with 5 years follow up they found that the pace of death was $(5.7 \%)$, MI (4.9\%) and clinical DTLR was (16.4\%), however for the crisis required for $\mathrm{CABG}$ was excluded from this investigation.

Likewise in study led by [13] the in medical clinic result was discovered that, the pace of death was $(0 \%)$, and MI $(0.9 \%)$, DTRL $(1.8 \%)$. be that as it may, with respect to long haul follow up was $(0.9 \%)$ demise , MI (3.7\%), and DTRL (4.6\%).

In [13] the creators directed that at year follow up there was $(10.3 \%)$ cardiovascular passing, ( $2.3 \%$ ) MI, $(0.0 \%)$ rising $\mathrm{CABG}$, and $(1.7 \%)$ for clinically DTLR.

We saw that in the previously mentioned examinations during the inpatient, one month and half year follow up the result was practically close to our investigation, and there was expanded pace of heart demise , MI , emanant CABG , and clinical DTLR with long haul follow up ( year -5 years $)$ in this investigations. 
Additionally we saw that the normal utilization of IVUS or OCT pre and post procedural as in [15] and OCT post procedural as in [16] not improving the result in contrast with our investigation.

\section{Conclusion}

In This study we found that it is safe, efficient and feasible to use self-expandable stent in patients presented with stable and acute coronary syndrome with atypical coronary anatomy regarding (in hospital stay, short and mid-term) clinical outcome.

\section{Recommendation}

From present study, in challenging anatomy such as tapered vessels, aneurysmal, ectatic and or large vessels, the Self-expandable stent could be used in patients with stable and acute coronary syndrome with considerable safety and feasibility regarding the in hospital, short and 6 month clinical outcome.

\section{Limitation}

- The self-expandable stent used, including the choice for stent was at the discretion of the operator.

- The angiograms were reviewed by a single expert only.

- No routine angiographic follow-up was performed in these patients.

- A matched control group with such atypical anatomical high-risk lesions treated with balloon-expandable stents was not available.

- Intermediate term follow-up

\section{References}

[1] E.M. Antman , D.T. Anbe , P.W Armstrong , ACC/AHA guidelines for the management of patients with ST-elevation myocardial infarction-executive summary: a report of the American College of Cardiology/American Heart Association Task Force on Practice Guidelines (Writing Committee to Revise the 1999 Guidelines for the Management of Patients with Acute Myocardial Infarction . erratum in J Am Coll Cardiol, Vol.45, PP. 1376,2005.

[2] P.D. Mcgrath , D.J. Malenka , D.E. Wennberg ,Changing outcomes in percutaneous coronary interventions: a study of 34,752 procedures in northern New England, 1990 to 1997. Northern New England Cardiovascular Disease Study Group. J Am Coll Cardiol,Vol.34,PP.674-680,1999.

[3] D.O. Williams, R. Holubkov, W. Yeh , Percutaneous coronary intervention in the current era compared with 1985-1986: the National Heart, Lung, and Blood Institute Registries. Circulation, Vol. 102, PP.2945-2951,2000.

[4] Nakazawa G . Pathological findings at bifurcation lesions: the impact of flow distribution on atherosclerosis and arterial healing after stent implantation. J Am Coll Cardiol. 2010;55(16):1679-87.

[5] A. Hoye , I . Iakovou , L. Ge, Long-term outcomes after stenting of bifurcation lesions with the "crush"technique: predictors of an adverse outcome. J Am Coll Cardiol, Vol.47,PP.19491958,2006.

[6] R. Mehran , G. Dangas , A.S. Abizaid ,Angiographic patterns of in-stent restenosis. classification and implications for long-term outcome. Circulation, Vol. 100, PP.1872-1878,1999.

[7] A. IJsselmuiden, S. Verheye , First report on the use of a novel self-expandable stent for treatment of ST elevation myocardial infarction. Catheter Cardiovasc Interv ,74(6), PP.850-854,2009.

[8] A.V. Finn, M. Joner, G. Nakazawa, Pathological correlates of late drug-eluting stent thrombosis: strut coverage as a marker of endothelialization. Circulation, Vol.115(18), PP.2435-2441,2007.

[9] R. Lang, L. Badano, V. Mor -Avi, Recommendations for Cardiac Chamber Quantification by Echocardiography in Adults: An Update from the American Society of Echocardiography and the European Association of Cardiovascular Imaging.J Am Soc Echocardiography, Vol.28, PP.1-3 9,2015.

[10] T. Nguyen, D. Hu, S. Chen, Angiographic views, Practical Handbook of Advanced Interventional Cardiology: Tips and Tricks, 4th Edition, Wiley Blackwell, Vol.2, PP. 7-51,2013.

[11] Robert-Jan, van Geuns, Katherin Awad, The Role of Self-expanding Stents in Patients with Atypical Coronary Anatomy . Interventional Cardiology Review, Vol. 9(1), PP.6-11,2014.

[12] C.G Bekker, M.J. Grun deken, P.WoudstraFiveyear clinical follow-up of the STENTYS selfapposing stent in complex coronary anatomy: a single-centre experience with report of specific angiographic indications, Netherlands Heart Journal, Vol. 26 (5), PP. 263-27,2018.

[13] Simona , Pierfrancesco , Luca Mariani , Cardiology Department, Mazzoni Hospital, Via degli Iris n. 1, Ascoli Piceno 63100, Italy, A real world single centre experience using the STENTYS self-expanding coronary stent, International journal of cardiology April , Vol.(15) ,PP. 209 Pages 57-59,2016.

[14] Luigi Emilio Pastormerlo, Marco Ciardetti a, Michele Coceani a, Self-expanding stent for complex percutaneous coronary interventions: A real life experience, Cardiovasc Revasc Med .April-May, Vol.17(3),PP. 186-189,2016.

[15] Giovanni Amoroso, MD, PhD; Robert-Jan van Geuns MD, PhD; Christian Spaulding, MD, PhD, Assessment of the safety and performance of the STENTYS self-expanding coronary stent in acute myocardial infarction: results from the APPOSITION I study., Euro Intervention, Vol.7, PP.428-436,2011.

[16] Robert Jan , Corrado Tamburino , Jean Fajader , APPOSITION II study : Self-expanding versus balloon-expandable stents in acute myocardial 
infarction, JACC Cardiovasc Interv.jcin, Vol.08, PP.16,2012.

[17] T. Koch Karel, J. Grundeken Maik, One-year clinical outcomes of the STENTYS SelfApposing® coronary stent in patients presenting with ST-segment elevation myocardial infarction: results from the APPOSITION III registry, journal of EuroPCR in collaboration with the Working Group on Interventional Cardiology of the
European Society of Cardiology - Euro Intervention , Vol.11, PP.264-271,2015.

[18] Robert-Jan M. van Geuns, Tuncay Yetgin , Alessio La Manna, APPOSITION IV study : STENTYS Self-Apposing® sirolimus-eluting stent in ST-segment elevation myocardial infarction. EuroIntervention, Vol. 11, PP.e1267e1274,2016. 